\title{
Resiliencia infantil y pensamiento multidimensional como factor de transformación social
}

Sandra Milena Alvarán López

Doctora en Cooperación Internacional Universidad de Antioquia - Colombia smalvaranl@gmail.com

https://orcid.org/oooo-0001-6867-3277

Cindy Tatiana Carrero Torres

Doctoranda en Desarrollo Local y Cooperación

Corporación Universitaria Minuto de Dios - Colombia

cindy.carrero@uniminuto.edu

https://orcid.org/0000-0003-1455-4488

Héctor Rafael Castellanos Triviño Magíster en Educación

Universidad Santo Tomás, CAU Villavicencio - Colombia

hectorcastellanos@ustadistancia.edu.co https://orcid.org/0000-0002-8592-2607

Heidy Yohanna Pinilla López

Doctoranda en Desarrollo Local y Cooperación

Corporación Universitaria Minuto de Dios - Colombia

hpinilla@uniminuto.edu

https://orcid.org/0000-0003-2036-6993

Artículo de investigación

Recepción: 15 de enero de 2019 Aprobación: 10 de marzo de 2019 https://doi.org/10.19053/22160159.110.n23.2019.9727

\section{Resumen}

La población de niños, niñas y adolescentes ha sido una de las principales víctimas del conflicto armado en Colombia. Afectaciones de orden físico, cognitivo y psicoafectivo son las principales consecuencias de la exposición de la infancia a una larga historia de violencia en el país. Ante este panorama, surge la intención de demostrar que cuando un niño, niña o adolescente participa en comunidades de diálogo, como escenarios de educación filosófica con perspectiva social, logra ser mucho más resiliente y tener mayores mecanismos de protección internos para enfrentarse a los factores de riesgo o marginalidad derivados del conflicto. Este artículo presenta los resultados de la investigación Promoción de la resiliencia en niños y niñas víctimas del conflicto armado, aportes desde las comunidades de diálogo, basada en el paradigma empírico analítico. El estudio es cuasiexperimental, con enfoque cuantitativo. Participaron niños, niñas y adolescentes hijos de familias víctimas del conflicto armado colombiano, habitantes de los municipios de Villavicencio, Meta, y Soacha, Cundinamarca.

Palabras clave: filosofía, infancia, conflicto armado, marginalidad, resiliencia 


\section{Resilience in children and multidimensional thinking as a social transforming factor}

\section{Abstract}

The child and adolescent populations have been some of the primary victims of the armed conflict in Colombia. Physical, cognitive, and psychoaffective damages are the major consequences of childhood exposure to a long history of violence in the country. Against this background, it is important to demonstrate that when a boy, a girl, or an adolescent participates in communities of inquiry as educational psychology scenarios with a social perspective, they become more resilient and they strengthen their internal protection mechanism for dealing with risk factors or marginalization caused by the conflict. This article presents the results of the research Promotion of resilience among children victims of the armed conflict, contributions from communities of inquiry, which was based on the empirical-analytical paradigm. This quasi-experimental study follows a quantitative approach. It involved children and adolescents from families that were victims of the Colombian armed conflict, inhabitants of the municipalities of Villavicencio, in Meta, and Soacha, in Cundinamarca.

Keywords: philosophy, childhood, armed conflict, marginalization, resilience

\section{La résilience des enfants et la pensée multidimensionelle comme un facteur de transformation sociale}

\section{Résumé}

La population infantile et adolescente a été l'une des principales victimes du conflit armé en Colombie. Les dommages d'ordre physique, cognitif, et psychoaffectif constituent les conséquences principales de l'exposition des enfants à une longue histoire de violence dans le pays. Dans ce contexte, il est important de démontrer que, lorsqu'un garçon, une fille, ou un adolescent participe à des communautés d'apprentissage en tant 
que scénarios d'éducation philosophique dans une perspective sociale, ils deviennent plus résilients et ils renforcent leurs mécanismes internes de protection pour faire face aux facteurs de risques et à la marginalisation résultant du conflit. Cet article présente les résultats de la recherche $L a$ promotion de la résilience parmi les enfants victimes du conflit armé, des contributions des communautés d'apprentissage, qui est fondé sur le paradigme empirique-analytique. L'étude intègre un modèle quasiexpérimental avec une approche quantitative. Il a réuni des enfants et des adolescents des familles victimes du conflit armé colombien, des habitants des municipalités de Villavicencio, à Meta et Soacha, à Cundinamarca.

Mots-clés : philosophie, enfance, conflit armé, marginalisation, résilience

\section{Resiliência infantil e pensamento multidimensional como fator de transformação social}

\section{Resumo}

A população de crianças e adolescentes tem sido uma das principais vítimas do conflito armado na Colômbia. Afetações de natureza física, cognitiva e psicoativa são as principais consequências da exposição da infância a uma longa história de violência no país. Neste panorama, surge a intenção de demonstrar que quando uma criança ou adolescente participa em comunidades de diálogo, como cenários de educação filosófica com uma perspectiva social, logra ser muito mais resiliente e ter maiores mecanismos de proteção interna para enfrentar os fatores de risco ou a marginalidade derivada do conflito. Este artigo apresenta os resultados da pesquisa Promoção da resiliência em crianças vítimas do conflito armado, contribuições desde as comunidades de diálogo, baseada no paradigma empírico analítico. O estudo é quase experimental, com uma abordagem quantitativa. Participaram meninos, meninas e adolescentes filhos de famílias vítimas do conflito armado colombiano, habitantes dos municípios de Villavicencio, Meta, e Soacha, Cundinamarca.

Palavras-chave: filosofia, infância, conflito armado, marginalidade, resiliência 
Sandra Milena Alvarán López • Cindy Tatiana Carrero Torres • Héctor Rafael Castellanos Triviño

- Heidy Yohanna Pinilla López

\section{Introducción}

El planteamiento de nuevos horizontes en la propuesta de Filosofía para niños [FpN] implica la consideración de aspectos ya propuestos en la obra de Matthew Lipman, en relación con los conceptos de comunidad de diálogo y pensamiento multidimensional, que si bien son fundamentales en la comprensión de la propuesta, para el contexto colombiano implican la adaptación de nuevas prácticas filosóficas que impacten de manera directa a la comunidad (Rojas, 2015). Es así que las comunidades de diálogo desde la educación filosófica con perspectiva social posibilitan el reconocimiento de nuevos escenarios en los que se aprende a pensar, desde la escucha atenta, el diálogo, el respeto por el pensamiento del otro y la construcción de nuevas alternativas de diálogo y comprensión de los problemas sociales, que, como el conflicto armado colombiano, requieren de acciones creativas para el abordaje de los efectos psicosociales que dejó en la población. Así mismo, la perspectiva social en esta propuesta implica realizar intervención en territorios en condiciones de marginalidad. Según datos de la Unidad para la atención y reparación integral a las víctimas (2019), a corte de junio del 2019 existen un registro total de 2.214.957 niños, niñas y adolescentes víctimas del conflicto armado en Colombia.

Por lo anterior, el proyecto de investigación denominado Promoción de la resiliencia en niños y niñas víctimas del conflicto armado, aportes desde las comunidades de diálogo - financiado por la Corporación Universitaria Minuto de Dios [Uniminuto], sede principal, y en alianza con la Universidad de Antioquia y la Universidad Santo Tomás, CAU Villavicencio-, se realiza en dos importantes municipios de Colombia: Soacha, el municipio con la mayor recepción de personas en condición de víctima del conflicto armado, y Villavicencio, capital de uno de los departamentos más golpeados por la violencia del país. La investigación supone que cuando los niños y las niñas participan en comunidades de diálogo, logran elevar sus niveles de resiliencia.

Para esta investigación, se entiende la resiliencia infantil como el resultado de dos variables: los factores de protección pensamiento multidimensional: crítico, creativo y cuidadoso- y los factores de riesgo - marginalidad asociada a las condiciones familiares y condiciones de pobreza del entorno-. 
Entre los factores de protección en la población infantil pueden encontrarse las capacidades personales. Para esta investigación se realizó la medición de 9 variables de estudio, de acuerdo con la propuesta de (Jaramillo, 2012), las cuales se cruzaron con las dimensiones del pensamiento multidimensional:

- Pensamiento crítico: autoestima, tareas adolescentes, pensamiento crítico.

- Pensamiento cuidadoso: asertividad, impulsividad, afectividad.

- Pensamiento creativo: adaptabilidad a situaciones nuevas y creatividad.

Los grupos de estudio participaron durante seis meses en comunidades de diálogo, entendidas como espacios que pretende[n] un mejoramiento del pensamiento, que se centra en buscar espacios para indagar, investigar, explorar y cuestionar los procesos que surgen en estas mismas actividades que se generan a partir de las discusiones, preguntas, juicios y razonamientos que los participantes van teniendo. (Zabala, 2014, p. 62)

En los grupos de Soacha, se implementó un currículo diseñado por el grupo Marfil de Uniminutollamado Cultivandopensamientos, una propuesta de educación filosófica con perspectiva social, que supone el desarrollo del pensamiento multidimensional. En Villavicencio, se desarrolló un currículo diseñado por el semillero de investigación Filosofía e Infancia de la Universidad Santo Tomás, basado en los presupuestos de FpN.

Durante el desarrollo de la investigación se evidenció que tanto en Soacha como en Villavicencio se presentan factores de riesgo para ser niño, niña y adolescente, asociados a dinámicas familiares y alta marginalidad en los territorios. Frente a las situación familiar se identificaron factores como la pérdida de seres queridos, nuevas tipologías y relaciones de poder coercitivas e impositivas, traducidas en violencia intrafamiliar y la tenue posibilidad de los padres, madres o adultos significativos de garantizar el cuidado y protección de los niños, niñas y adolescentes, debido a las condiciones del mercado laboral - trabajo informal, largas jornadas laborales, lejanía de las zonas de trabajo, etc.-, lo que limita la posibilidad de compartir tiempos significativos. En la mayoría de los casos, los niños y niñas asumen roles de cuidadores. 
Tanto Soacha como en Villavicencio se sufre una fragilidad institucional y falta de recursos. La consecuencia más inmediata es la ausencia de servicios públicos - hospitales, colegios, medios de transporte, entre otros-. Además, las instituciones realizan el presupuesto de sus proyectos con base en una población mucho menor a la que en realidad se debe atender.

El 93,3\% de los hogares de los niños y niñas participantes han sido afectados por abandono o despojo forzado de tierras; el 53,3\% ha sido afectado por amenazas; el $33,3 \%$ por actos terroristas, atentados, combates, enfrentamientos y hostigamientos; el 13,3\% por desaparición forzada; el $13,3 \%$ por homicidio; el 13,3\% por masacre; el $6,7 \%$ por tortura y el $6,7 \%$ por vinculación de menores de edad en actividades relacionadas con grupos armados. Asimismo, el $72,1 \%$ de la población muestra de la investigación pertenece al género femenino y el 27,9\% restante al masculino.

Por otro lado, de acuerdo con la composición familiar, se puede decir que el 50\% de los niños y niñas de la muestra viven con 30 4 personas; el 22,1\%, con 5 personas; el 14,7\%, con 2 personas; el $8,8 \%$, con 6 personas; y el 4,5\%, con 1 u 8 personas. El 50\% vive con hermanos; el $35,5 \%$, con padre y madre; el $26,5 \%$, con padrastro y madre; el 20,6\%, solo con la madre; el 10,3\%, con abuelo y/o abuela; el $8,8 \%$, con otro acompañante; el $4,4 \%$, con madrastra y padre; y el $1,5 \%$, solo con padre. A su vez, 6 niños, niñas y adolescentes viven con otro acompañante; dos niñas viven con madre sustituta, 33,3\%, por ser huérfanas de padre y madre; 4 viven solamente con los tíos, $66,6 \%$.

Lo anterior lleva a construir y reforzar imaginarios complejos de exclusión y discriminación social, que limitan la construcción de futuro de niños, niñas y adolescentes, además de naturalizar expresiones de violencia como forma de relacionamiento válida y la eliminación del otro - el par, el vecino, el amigo, el que piensa diferente-, como medio ejemplar para resolver los conflictos cotidianos.

\section{Método}

El proyecto de investigación Promoción de la resiliencia en NNA, víctimas del conflicto armado se basó en el paradigma empírico analítico, de tipo cuantitativo con aportes cualitativos, donde se 
aplicó un pre-test y un pos-test. Del mismo modo, se planteó un diseño cuasi experimental, en aras de una perspectiva correlacional de variables en el marco del proyecto, frente a las diversas características de los presentes contextos marginales. Según lo anterior, la línea investigativa refiere al análisis y contraste entre las diferencias y similitudes halladas en los procesos ejercidos en cada territorio, respecto a las comunidades de diálogo, teniendo como referencia el distintivo cultural evidenciado en cada uno de los contextos.

La muestra poblacional fue intencionada. Se definieron dos grupos, los cuales cumplieron con las siguientes características: niños, niñas y adolescentes en edades de 9 a 14 años, escolarizados. Para Villavicencio, se definió un grupo de 60 niñas, niños y adolescentes, y para Soacha, un grupo de 90 participantes.

Para la medición de la resiliencia se utilizó la escala Likert JJ63 construida por Jaramillo (2012), docente investigador de la Facultad de Ciencias Médicas de la Universidad de Cuenca. Consiste en una serie de situaciones que pueden ocurrir o no en la persona respecto del medio ambiente, externo e interno, para un total de 63 ítems; corresponden 7 preguntas a cada uno de los 9 ejes que mide el instrumento.

Las variables cuantitativas se describieron con sus valores mínimo, máximo, promedio y desviación estándar o mediana acompañada del rango intercuartílico, dependiendo de la distribución de los datos previos a la prueba de normalidad. Las variables cualitativas se describieron por medio de medidas de frecuencias absolutas y relativas. Las distribuciones de frecuencias realizadas estarán acompañadas de intervalos de confianza binomial exacto del 95\%.

En el análisis estadístico, primero se comprobó la normalidad de los puntajes para cada una de las dimensiones, pre-test y posttest, mediante la prueba de Shapiro Wilk. Cuando los puntajes, pre-test y post-test, en las dimensiones, provenían de una población con distribución normal, se construyeron intervalos de confianza del 95\% para las diferencias de promedios en muestras relacionadas y se calcularon los tamaños del efecto, mediante la siguiente expresión (Sánchez-Meca, Marín-Martínez, \& López-

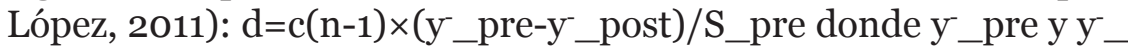


post son los promedios del puntaje en el pre-test y en el post-test respectivamente, y S_pre es la desviación estándar en el pre-test; $c(n-1)$ es el factor de corrección con $c(n-1)=1-3((4 \times n-5))$. Cuando los puntajes no cumplieron el supuesto de normalidad, se recurrió, para valorar las diferencias de los rangos promedios, a la prueba no paramétrica de Wilcoxon, acompañado del tamaño del efecto no paramétrico mediante la siguiente expresión (Dasborough, 2007): d=(Q_post-Q_pre)/S_bip , donde Qpost y Qpre son las medianas en el post-test y pre-test respectivamente, y Sbip es la desviación estándar biponderada del grupo de control para cada dimensión en el pre-test. Los valores del tamaño del efecto se tomaron en valor absoluto y la interpretación de ellos se hizo siguiendo la propuesta de Cohen (1988) y Grissom \& Kim (2005). También, se utilizó la prueba de McNemar para valorar la significación de los cambios de las categorías de la resiliencia antes y después de la intervención.

El procesamiento de la información se realizó en el programa estadístico SPSS versión 22 y el programa Epidat versión 3.1. Otros programas utilizados en el procesamiento son los de Microsoft Office como el Excel, Word y Power Point.

\section{Resultados}

A partir de la implementación del instrumento JJ63 al total de participantes seleccionados, desde el análisis realizado a los datos encontrados en la medición de pre-test y pos-test, identificamos los siguientes resultados por municipios, y en comparativo, así:

\section{Resultados de la medición de resiliencia en el grupo de estudio de Villavicencio.}

La resiliencia presentó la media más alta de los puntajes obtenidos en el puntaje inicial con un 221,0, desviación estándar de 22,1. La prueba estadística de normalidad de Kolmogorov-Smirnov evidencia que el valor de $\mathrm{p}^{*}$ para el puntaje inicial es superior al alfa como se observa en la tabla 1, por lo tanto, no se rechaza la hipótesis nula y se concluye que los datos de la resiliencia no siguen una distribución normal, aspecto coherente con las características específicas de la población participante. 
El tamaño del efecto de la resiliencia, de 0,02, se catalogó como baja, esto es, los puntajes de resiliencia, después de las comunidades de diálogo, fueron superiores que los puntajes si no se hubiera llevado a cabo la intervención, en el 50\% de las comparaciones, según Cohen (1988) y Grissom (1994).

\section{Tabla 1}

Mediana y variabilidad de la Resiliencia pre-test y post-test y valoración estadística de la diferencia y del tamaño del efecto en Villavicencio, 2018

\begin{tabular}{llllll}
\hline Puntaje & $\mathbf{p}^{*}$ & Mediana & D.E & P** $^{*}$ & D \\
\hline Antes de la intervención & 0,003 & 221,0 & 22,1 & & \\
Después de la intervención & 0,200 & 220,5 & 26,1 & 0,417 & 0,02 \\
\hline
\end{tabular}

Nota: ${ }^{*} \mathrm{p}^{*}$ : Prueba de Kolmogorov-Smirnov; $\mathrm{p}^{* *}$ : Prueba no paramétrica de Wilcoxon; d: Tamaño del efecto. Fuente. Elaboración propia, 2019.

\section{Resultados de la medición de resiliencia en el grupo de estudio de Soacha.}

La resiliencia presentó la media más alta de los puntajes obtenidos en el puntaje inicial con un 216, desviación estándar de 19. La prueba estadística de normalidad de Kolmogorov-Smirnov evidencia que el valor de $\mathrm{p}^{*}$ para el puntaje inicial es superior al alfa, por lo tanto, los datos de la resiliencia siguen una distribución normal. Una vez se identifica el proceso de normalidad se realiza la prueba estadística de $t$ student para muestras relacionadas, dado que siguen una distribución normal, donde el valor de $\mathrm{p}^{* *}$ permite verificar diferencias significativas entre los puntajes inicial y final, observando el valor de probabilidad, al estar por debajo de 0,05 concretamente igual a 0,045 . El tamaño del efecto de la resiliencia, de 0,411, se catalogó como Baja, esto es, los puntajes de resiliencia, después de las comunidades de diálogo fueron superiores que los puntajes si no se hubiera llevado a cabo la intervención, en el 50\% de las comparaciones, según Cohen (1988) y Grissom (1994). 
Sandra Milena Alvarán López • Cindy Tatiana Carrero Torres • Héctor Rafael Castellanos Triviño - Heidy Yohanna Pinilla López

Tabla 2

Mediana y variabilidad de la Resiliencia pre-test y post-test y valoración estadística de la diferencia y del tamaño del efecto en

Soacha, 2018

\begin{tabular}{|c|c|c|c|c|c|c|c|}
\hline \multirow{2}{*}{ Puntaje } & \multirow{2}{*}{$\mathrm{p}^{*}$} & \multirow{2}{*}{ Media } & \multirow{2}{*}{ D.E } & \multicolumn{2}{|c|}{ IC 95\% $\left(\mu_{1}-\mu_{2}\right)^{* * *}$} & \multirow{2}{*}{$\mathrm{P}^{* * * *}$} & \multirow{2}{*}{ d } \\
\hline & & & & Inferior & Superior & & \\
\hline Antes de la intervención &, 560 & 216,36 & 19,028 & \multirow{2}{*}{0,168} & \multirow{2}{*}{15,473} & \multirow{2}{*}{0,045} & \multirow{2}{*}{0,411} \\
\hline Después de la intervención & 807 & 208,54 & 22,570 & & & & \\
\hline
\end{tabular}

Nota: * $\mathrm{p}^{*}$ : Prueba de Kolmogorov-Smirnov; $\mathrm{p}^{* *}$ : Diferencia de promedios poblacionales; $\mathrm{p}^{* * * *}$ : Prueba t para muestras relacionadas; d: Tamaño del efecto. Fuente. Elaboración propia, 2019.

\section{Comparativo en la medición de resiliencia en los grupos de estudio de Villavicencio y Soacha.}

Asumiendo una distribución normal y varianzas iguales entre las diferentes dimensiones del instrumento JJ63, se calcula el estadístico $\mathrm{T}$ para muestras independientes. Se observa que en la recolección inicial se presentaron diferencias significativas únicamente para la dimensión afectividad, favoreciendo y con promedio más alto para Soacha, estadístico de 0,007. El intervalo de confían del 95\% comprueba la existencia de diferencias significativas entre 0,5 y 3,7 puntos, valores que no incluyen el valor de 0 , como se ve en la tabla 3 .

Tabla 3

Media y variabilidad de la resiliencia pre-test y valoración estadística de la diferencia a través de prueba ten Soacha y Villavicencio, 2018

\begin{tabular}{|c|c|c|c|c|c|c|c|}
\hline \multirow{2}{*}{ Dimensión } & \multirow{2}{*}{ Ciudad } & \multirow[t]{2}{*}{$\mathbf{N}$} & \multirow{2}{*}{ Media } & \multirow{2}{*}{$\begin{array}{l}\text { Desviación } \\
\text { estándar }\end{array}$} & \multirow{2}{*}{$\begin{array}{c}{ }^{*} \text { Pruebat } \\
\text { para igualdad } \\
\text { de medias }\end{array}$} & \multicolumn{2}{|c|}{$\begin{array}{c}{ }^{* *} \text { IC } 95 \% \text { de la } \\
\text { diferencia }\end{array}$} \\
\hline & & & & & & Inferior & Superior \\
\hline \multirow{2}{*}{$\begin{array}{c}\text { Funcionalidad } \\
\text { Familiar }\end{array}$} & Villavicencio & 74 & 26,34 & 4,05 & \multirow{2}{*}{ 0,093 } & \multirow{2}{*}{$-0,236$} & \multirow{2}{*}{3,014} \\
\hline & Soacha & 39 & 24,95 & 4,32 & & & \\
\hline \multirow{2}{*}{ Autoestima } & Villavicencio & 74 & 24,03 & 3,27 & \multirow{2}{*}{0,550} & \multirow{2}{*}{$-0,890$} & \multirow{2}{*}{1,662} \\
\hline & Soacha & 39 & 23,64 & 3,22 & & & \\
\hline \multirow{2}{*}{ Asertividad } & Villavicencio & 74 & 22,08 & 4,38 & \multirow{2}{*}{0,093} & \multirow{2}{*}{$-3,107$} & \multirow{2}{*}{0,244} \\
\hline & Soacha & 39 & 23,51 & 4,06 & & & \\
\hline \multirow{2}{*}{$\begin{array}{c}\text { Adaptabilidad } \\
\text { a situaciones } \\
\text { nuevas }\end{array}$} & Villavicencio & 74 & 23,15 & 4,85 & \multirow[b]{2}{*}{0,418} & \multirow[b]{2}{*}{$-2,572$} & \multirow[b]{2}{*}{1,075} \\
\hline & Soacha & 39 & 23,90 & 4,25 & & & \\
\hline \multirow{2}{*}{$\begin{array}{c}\text { Tareas } \\
\text { adolescentes }\end{array}$} & Villavicencio & 74 & 24,91 & 4,47 & \multirow{2}{*}{0,558} & \multirow{2}{*}{$-1,113$} & \multirow{2}{*}{2,052} \\
\hline & Soacha & 39 & 24,44 & 3,02 & & & \\
\hline
\end{tabular}




\begin{tabular}{|c|c|c|c|c|c|c|c|}
\hline \multirow{2}{*}{ Creatividad } & Villavicencio & 74 & 24,07 & 5,48 & \multirow{2}{*}{0,765} & \multirow{2}{*}{$-1,676$} & \multirow{2}{*}{2,273} \\
\hline & Soacha & 39 & 23,77 & 4,06 & & & \\
\hline \multirow{2}{*}{$\begin{array}{l}\text { Capacidad de } \\
\text { pensamiento } \\
\text { crítico }\end{array}$} & Villavicencio & 74 & 22,23 & 4,23 & \multirow[b]{2}{*}{0,926} & \multirow[b]{2}{*}{$-1,729$} & \multirow[b]{2}{*}{1,573} \\
\hline & Soacha & 39 & 22,31 & 4,16 & & & \\
\hline \multirow{2}{*}{ Impulsividad } & Villavicencio & 74 & 25,55 & 5,18 & \multirow{2}{*}{0,504} & \multirow[b]{2}{*}{$-1,332$} & \multirow{2}{*}{2,697} \\
\hline & Soacha & 39 & 24,87 & 5,05 & & & \\
\hline \multirow{2}{*}{ Afectividad } & Villavicencio & 74 & 22,80 & 4,09 & \multirow{2}{*}{0,007} & \multirow{2}{*}{$-3,756$} & \multirow{2}{*}{$-0,598$} \\
\hline & Soacha & 39 & 24,97 & 3,91 & & & \\
\hline \multirow{2}{*}{$\begin{array}{l}\text { Resiliencia } \\
\text { general }\end{array}$} & Villavicencio & 74 & 215,15 & 22,15 & \multirow{2}{*}{0,773} & \multirow{2}{*}{$-9,496$} & \multirow{2}{*}{7,076} \\
\hline & Soacha & 39 & 216,36 & 19,03 & & & \\
\hline
\end{tabular}

Nota: *se asume varianza iguales y distribución normal, * Prueba t para igualdad de medias, **intervalo de confianza de la diferencia. Fuente: Elaboración propia, 2019.

Luego de realizar las comunidades de diálogo se presentan diferencias significativas en 3 de las variables del instrumento JJ63, donde la dimensión de tareas adolescentes, creatividad y capacidad de pensamiento crítico presentan un aumento y una mejora considerable en Villavicencio en comparación con Soacha, lo comprueban los valores de (0,006 tareas adolescentes), (0,002 creatividad) y (0,003 pensamiento crítico). Las tres dimensiones con cambios significativos presentan intervalos de confianza que no incluyen el valor y presenta cambios en la mediana de la siguiente manera:

Pensamiento crítico presentó en Soacha 21,33 y en Villavicencio 24,27; creatividad presentó en Soacha 22,36 y en Villavicencio 25,42; tareas adolescentes presentó en Soacha 23,79 y en Villavicencio 26,51; estas diferencias fueron significativas a favor de Villavicencio, donde las comunidades de diálogo presentaron un mayor impacto en estas dimensiones en comparación con Soacha (tabla 4).

Tabla 4.

Media y variabilidad de la resiliencia post-test y valoración estadística de la diferencia a través de prueba ten Soacha y Villavicencio, 2018

\begin{tabular}{|c|c|c|c|c|c|c|c|}
\hline \multirow[t]{2}{*}{ Dimensión } & \multirow[t]{2}{*}{ Ciudad } & \multirow[t]{2}{*}{$\mathbf{N}$} & \multirow[t]{2}{*}{ Media } & \multirow{2}{*}{$\begin{array}{c}\text { Desviación } \\
\text { estándar }\end{array}$} & \multirow{2}{*}{$\begin{array}{c}\text { * Prueba t } \\
\text { para igualdad } \\
\text { de medias }\end{array}$} & \multicolumn{2}{|c|}{$\begin{array}{l}{ }^{* *} \text { IC 95\% de la } \\
\text { diferencia }\end{array}$} \\
\hline & & & & & & Inferior & Superior \\
\hline \multirow{2}{*}{$\begin{array}{c}\text { Funcionalidad } \\
\text { Familiar }\end{array}$} & Villavicencio & 74 & 24,69 & 3,98 & \multirow{2}{*}{0,575} & \multirow{2}{*}{$-1,987$} & \multirow{2}{*}{1,109} \\
\hline & Soacha & 39 & 25,13 & 3,89 & & & \\
\hline \multirow{2}{*}{ Autoestima } & Villavicencio & 74 & 23,22 & 4,63 & \multirow{2}{*}{0,337} & \multirow{2}{*}{$-2,631$} & \multirow{2}{*}{,910 } \\
\hline & Soacha & 39 & 24,08 & 4,28 & & & \\
\hline \multirow{2}{*}{ Asertividad } & Villavicencio & 74 & 22,68 & 5,15 & \multirow{2}{*}{0,607} & \multirow{2}{*}{$-1,410$} & \multirow{2}{*}{2,403} \\
\hline & Soacha & 39 & 22,18 & 4,25 & & & \\
\hline
\end{tabular}


Sandra Milena Alvarán López • Cindy Tatiana Carrero Torres • Héctor Rafael Castellanos Triviño - Heidy Yohanna Pinilla López

\begin{tabular}{|c|c|c|c|c|c|c|c|}
\hline \multirow{2}{*}{$\begin{array}{l}\text { Adaptabilidad } \\
\text { a situaciones } \\
\text { nuevas }\end{array}$} & Villavicencio & 74 & 22,81 & 4,47 & \multirow{2}{*}{0,611} & \multirow{2}{*}{$-1,302$} & \multirow{2}{*}{2,206} \\
\hline & Soacha & 39 & 22,36 & 4,48 & & & \\
\hline \multirow{2}{*}{$\begin{array}{c}\text { Tareas } \\
\text { adolescentes }\end{array}$} & Villavicencio & 74 & 26,51 & 5,30 & \multirow{2}{*}{0,006} & \multirow{2}{*}{, 785} & \multirow{2}{*}{4,652} \\
\hline & Soacha & 39 & 23,79 & 4,13 & & & \\
\hline \multirow{2}{*}{ Creatividad } & Villavicencio & 74 & 25,42 & 4,97 & \multirow{2}{*}{0,002} & \multirow{2}{*}{1,191} & \multirow{2}{*}{4,929} \\
\hline & Soacha & 39 & 22,36 & 4,35 & & & \\
\hline \multirow{2}{*}{$\begin{array}{l}\text { Capacidad de } \\
\text { pensamiento } \\
\text { crítico }\end{array}$} & Villavicencio & 74 & 24,27 & 5,18 & \multirow[b]{2}{*}{0,003} & \multirow[b]{2}{*}{1,029} & \multirow[b]{2}{*}{4,845} \\
\hline & Soacha & 39 & 21,33 & 4,21 & & & \\
\hline \multirow{2}{*}{ Impulsividad } & Villavicencio & 74 & 25,24 & 5,66 & \multirow{2}{*}{0,118} & \multirow{2}{*}{,- 445} & \multirow{2}{*}{3,906} \\
\hline & Soacha & 39 & 23,51 & 5,32 & & & \\
\hline \multirow{2}{*}{ Afectividad } & Villavicencio & 74 & 23,15 & 4,52 & \multirow[b]{2}{*}{0,491} & \multirow[b]{2}{*}{$-2,501$} & \multirow{2}{*}{1,209} \\
\hline & Soacha & 39 & 23,79 & 5,11 & & & \\
\hline \multirow{2}{*}{$\begin{array}{l}\text { Resiliencia } \\
\text { general }\end{array}$} & Villavicencio & 74 & 217,99 & 26,16 & \multirow{2}{*}{0,059} & \multirow{2}{*}{,- 352} & \multirow{2}{*}{19,248} \\
\hline & Soacha & 39 & 208,54 & 22,57 & & & \\
\hline
\end{tabular}

Nota: * se asume varianza iguales y distribución normal. * Prueba t para igualdad de medias, **intervalo de confianza de la diferencia. Fuente: Elaboración propia, 2019.

\section{Discusión}

La resiliencia puede definirse como la capacidad y el proceso que surge en cada persona cuando se enfrenta a situaciones que puedan representar adversidad o vulnerabilidad, y frente a ellas, el individuo se apoya en distintas habilidades o recursos internos y externos, que permiten que este pueda considerarse capaz de enfrentar y trascender de una forma positiva, ante el momento estresor en concreto. De aquí, que la resiliencia se entienda como una potencialidad y una posibilidad universal que permite a grupos, personas y comunidades hacer frente a las adversidades, al utilizar sus aptitudes personales como un escudo protector.

Amar, Kotliarenko \& Abello (2003), retomando a Grotberg, exponen que "la resiliencia es parte del proceso evolutivo y debe ser promovida desde la niñez. Así, vemos cómo se comienza a plantear la importancia de los factores protectores para el desarrollo sano de la infancia" (p. 166), permitiendo precisar que dichos factores o mecanismos de protección permiten que el niño o niña desarrolle potencialidades con las que pueda salir fortalecido ante situaciones que podrían considerarse aniquiladoras, y a su vez, pueda efectuar un ejercicio de enfrentamiento efectivo y adaptabilidad como una fuerza motivacional significativa de superación. 
Para Domínguez-de la Ossa \& García-Vesga (2013) las distintas definiciones de resiliencia enfatizan en características de los sujetos tales como: habilidad, adaptabilidad, baja susceptibilidad, enfrentamiento efectivo, capacidad, competencia, resistencia a la destrucción, conductas vitales positivas, temperamento especial y habilidades cognitivas, todas desplegadas frente a situaciones estresantes que les permiten superarlas. También se destaca que la resiliencia se desarrolla en función de procesos sociales e intrapsíquicos; está en función de los procesos interaccionales entre las personas y estos, a su vez, aportan a la construcción del sistema intrapsíquico del ser humano (Domínguez-de la Ossa \& GarcíaVesga, 2013).

Por otra parte, se encuentran los factores de riesgo en población infantil, entre los cuales se resalta la sobrecarga de responsabilidades, maltrato físico y psicológico, falta de atención médica, marginación y situación de pobreza extrema. Los niños que están en mayor situación de riesgo son aquellos expuestos a factores como dificultades económicas, enfermedad mental de alguno de los padres, prácticas de crianza inadecuadas, abuso, conflicto familiar o armado; es decir, un contexto material y psicosocial empobrecido(Fergunson \& Horwoord , 2003).

Los resultados arrojados por esta investigación demuestran que las comunidades de diálogo lograron diferencias significativas en 3 de 9 variables en estudio, como lo son tareas adolescentes, creatividad y capacidad de pensamiento crítico, presentando un aumento y una mejora considerable en Villavicencio en comparación con Soacha.

Sin embargo, se encontró que los factores de riesgo siguen siendo una talanquera al momento de hablar de la resiliencia, puesto que en los grupos de estudio se encontró que el 80,9\% de los niños, niñas y adolescentes participantes de la investigación en Villavicencio se reconocen como víctimas del conflicto, mientras que los niños y niñas del grupo de Soacha no se identifican como víctimas, sino que reconocen a su familia como tal. A esto se suma que el $83,3 \%$ de la muestra de Villavicencio tiene como lugar de procedencia el departamento de Meta: el 33,8\% pertenece al estrato 2, mientras que el $28,4 \%$ pertenece al estrato 1 . Por su parte, el 33\% de los niños de la muestra de Soacha tiene como lugar de procedencia Soacha y sus familias fueron las que migraron años 
Sandra Milena Alvarán López • Cindy Tatiana Carrero Torres • Héctor Rafael Castellanos Triviño - Heidy Yohanna Pinilla López

atrás: el $41 \%$ pertenece al estrato 2, mientras que el $59 \%$ pertenece al estrato 1.

Por su parte, Soacha se encuentra permeada por múltiples variables sociales, económicas y culturales que, al conjugarse con factores de violencia, actividades ilícitas, carencia de garantía de educación y oportunidades de sostenimiento, se constituyen como un obstáculo para el ejercicio de derechos de los niños y niñas. En este sentido, es común encontrar población procedente de varias regiones del país como Chocó, Quibdó, Tumaco, la Guajira, Tolima, Meta, Santanderes, Eje cafetero, entre otros; población que, por condiciones del conflicto armado, se vio obligada a migrar de sus territorios natales e instaurarse en zonas como Soacha. Han incrementado la población en barrios como Altos de la Florida y Altos de Cazucá.

Lo anterior demuestra que el desarrollo de comunidades de diálogo puede aumentar en alguna medida las habilidades resilientes de los niños y niñas y, por ende, fomentar en ellos y ellas sus mecanismos de protección, puesto que al generar procesos de desarrollo de pensamiento multidimensional hay una relación directa con las variables de resiliencia infantil. Cuando un niño y una niña logran pensar de manera crítica, creativa y cuidadosa su vida, su entorno, y hace una lectura de realidad diferente, puede llevar a mejorar su capacidad de enfrentar y sobreponerse a situaciones estresoras.

Sin embargo, los niños y las niñas víctimas del conflicto armado en los territorios de estudio sufren una fragilidad institucional y falta de recursos. Además las instituciones realizan el presupuesto de sus proyectos con base en una población mucho menor a la que en realidad se debe atender, es así como se evidencia tal como lo señalan Angarita, Andrade, Perico, Henao y Zuluaga (2011).

Algo más de la mitad de las familias desplazadas en Colombia viven en condiciones de hacinamiento crítico [...] en un mismo cuarto duermen hasta tres personas y el $37 \%$ de los hogares de desplazados no tiene acceso a todos los servicios públicos, lo que muestra que una altísima proporción de la población desplazada ocupada estaría en la informalidad. (Garay citado por Angarita et al. 2011, p. 53)

La condición de las familias suele ser tan precaria que el $26 \%$ de los niños menores de 5 años están desnutridos y el 7\%, pese a estar 
diagnosticados, no ha recibido ninguna atención médica(Angarita et al. 2011, p. 53).

En este marco, los niños, niñas y adolescentes se ven enfrentados en su diario vivir a constantes factores de riesgo, inmersos en las lógicas territoriales, familiares y personales, en donde las comunidades de diálogo actuaron como espacio protector frente a tales contextos marginales. Así pues, mediante las comunidades de diálogo, se evidenció el aumento de las capacidades de pensamiento crítico, lo cual visibilizó que los partícipes de las sesiones se muestran más conscientes de su realidad y del papel que juegan, en la construcción y transformación de un mejor entorno social.

Del mismo modo, se evidenciaron grandes avances referentes a la manera de participación de los niños y adolescentes frente a las comunidades de diálogo como herramienta propia del proyecto ejecutado, pues, más allá de los temas determinados, se evidenciaba una apertura a temas encaminados hacia la realidad que viven los niños y adolescentes. El diálogo fue un puente que permitió tocar temas personales y un encuentro de desahogo y socialización entre compañeros y pares.

Desde esta perspectiva, se comprende que los participantes hacen una movilización subjetiva para lograr legitimar a los otros y otras como interlocutores válidos en el establecimiento del diálogo, la construcción de conocimiento y la transformación de relaciones para avanzar en la garantía de sus derechos, lo cual permite que gesten una conciencia política que ilustre una capacidad de agencia individual y colectiva, encaminada a miradas críticas de empoderamiento, apropiación del territorio y búsqueda de justicia.

Lo anterior evidencia que el ejercicio de las comunidades de diálogo trasciende las dimensiones del espacio de un aula o una herramienta magistral y netamente estructural. Por el contrario, representa un impacto multidimensional en las distintas formas de ser y estar en el territorio, en donde se promueve que los niños y niñas descubran

otras alternativas que les ayudan a pensar los problemas y sus posibles soluciones, logran poco a poco pensar por sí mismos, también, confrontar, valorar y revaluar sus ideas y las de los demás, buscando siempre el respeto y las opiniones diversas. (Zabala, 2014, p. 62) 
Sandra Milena Alvarán López • Cindy Tatiana Carrero Torres • Héctor Rafael Castellanos Triviño • Heidy Yohanna Pinilla López

Ello permite romper con las lógicas marcadas históricamente por el miedo o las verdades lapidarias, que intentan corroer el pensamiento de los niños y niñas, por lo tanto se puede evitar repetir o seguir reproduciendo círculos de pobreza y miseria heredados tras generaciones y apostar por alternativas en los proyectos de vida que permitan generar nuevas formas de vida, si se trabaja en el desarrollo de pensamiento crítico, creativo y cuidadoso.

En este sentido, la resiliencia es un factor clave para transformar no solo el análisis del contexto, sino para mitigar las vulnerabilidades en la medida en que se construyen y reconstruyen los imaginarios sociales, las creencias arraigadas y las relaciones que por causa del conflicto fueron fracturadas, debilitadas o por el contrario eran inexistentes.

De igual forma, las relaciones que surgían en las comunidades de diálogo se presentaban de manera recíproca y tenían en cuenta la validación de los diferentes saberes tanto de los niños y adolescentes partícipes, como de los facilitadores, en alusión a principios de la educación popular, la cual plantea que el educador no es el único poseedor de conocimiento, sino que los saberes son una construcción y reconstrucción de manera conjunta y constante con los educandos.

En efecto, los niños, niñas y adolescentes partícipes de las comunidades de diálogo, potenciaron habilidades y capacidades resilientes, como la creatividad. De manera autónoma y consciente, se gestaron nuevas ideas que contribuyeron no solo a su ambiente escolar, sino que repercutieron en sus posturas frente al territorio y su propia vida. El pilar fundamental fueron los talentos y potencialidades con los que cuentan, para así promover un estilo de vida distinto al del imaginario social que pretende gestar alternativas que aporten a la transformación. La creatividad, como mecanismo de protección, puede determinarse como un escape o herramienta, para la condición de vulnerabilidad presente.

Asimismo, para entender la realidad social de los niños, niñas y adolescentes se debe tener en cuenta que solo se puede comprender a un individuo dentro del contexto social en el que vive, como integridad holística donde cada dimensión está interconectada e incide en la vida cotidiana de los mismos. Por esta razón, no se deben estandarizar las comunidades de diálogo, sino que se deben focalizar y adaptar a las dinámicas y particularidades del contexto social y cultural del territorio. Se constituyen en una invitación a 
pensar en la multiplicidad presente en sus construcciones identitarias y subjetivas y sus agentes relacionales como lo son la familia, la escuela y la comunidad. Esta invitación parte del reconocimiento de que dichas subjetividades e identidades se construyen en los significados compartidos. (Fajardo, Ramírez, Valencia \& Ospina, 2018, p. 12)

Así lo propone la educación filosófica en perspectiva social, para, de esta forma, generar una transformación social de mayor impacto. También, es imperante comprender la dimensión cultural como un entramado de valores, percepciones, intereses y significaciones de una comunidad, que permean las diversas esferas de la vida cotidiana de los sujetos sociales.

La cultura juega el papel principal al momento de entender e intervenir en las realidades y contextos, pues determina el porqué del accionar humano y las diversas particularidades de cada población. De este modo, se fortalecen procesos de empoderamiento participativo y democrático, que sirven de base para la promoción de cohesión y articulación social, en donde a partir del desarrollo del pensamiento en articulación con las apuestas de la resiliencia, se configuren escenarios para la transformación y encuentro con el

conocimiento y comprensión de la realidad [...] en donde se hace pertinente pensar en una enseñanza filosófica que traspase un acumulado de teorías y postulados para hacerla un principio de vida, es decir, hacer filosofía es enseñar a los otros y a sí mismo a pensar mejor. (Suárez, Gonzalez \& Lara , 2016, p. 241)

Es a partir del reconocimiento de los agentes internos, de sus diversas necesidades, potencialidades, carencias y oportunidades, que pueden gestarse soluciones acordes a sus realidades y problemas.

\section{Referencias}

Amar, J., Kotliarenko, M., \& Abello, R. (2003). Factores psicosociales asociados con la resiliencia en niños colombianos víctimas de violencia intrafamiliar. Investigación \& Desarrollo, 11(1), 162-197. Recuperado de http://www.redalyc.org/articulo.oa?id=26811107

Angarita, L., Andrade, J., Perico, L., Henao, N., \& Zuluaga, Y. (2011). Desplazamiento Forzado y conflicto armado. Niños y niñas vulnerados en sus derechos humanos. Orbis. Revista Científica de Ciencias Humanas, 7(20), 51-78. Recuperado de http://www.redalyc.org/ articulo.oa?id=70922149003 
Sandra Milena Alvarán López • Cindy Tatiana Carrero Torres • Héctor Rafael Castellanos Triviño

- Heidy Yohanna Pinilla López

Cohen, J. (1988). Statistical Power Analysis for the Behavioral Sciences (2nd ed.). NJ: Hillsdate.

Dasborough, M. (2007). Book Review: Effect Sizes for Research: A Broad Practical Approach. Mahwah, NJ: Lawrence Erlbaum. Organizational Research Methods, 1O(3), 542-545. https://doi. org/10.1177/1094428106295495

Domínguez-de la Ossa, E., \& García-Vesga, M. (2013). Desarrollo teórico de la Resiliencia y su aplicación en situaciones adversas: Una revisión analítica. Revista Latinoamericana de Ciencias Sociales, Niñez y Juventud, 11(1), 63-77. Recuperado de http://www.redalyc.org/ articulo.oa?id=77325885001

Fajardo, M., Ramírez, M., Valencia, M., \& Ospina, M. (2018). Más allá de la victimización de niñas y niños en contextos de conflicto armado: potenciales para la construcción de paz. Universitas Psychologica, 17(1), 1-14. https://doi.org/10.11144/Ja veriana.upsy17-1.mavn

Fergunson, D., \& Horwoord, J. (2003). Resilience and Vulnerability: Adaptation in the Context of Childhood Adversities. Cambridge, EEUU: Cambridge University Press

Grissom, R. (1994). Probability of the superior outcome of one treatment over another. Journal of Applied Psychology, 79(2), 314-316

Grissom, R., \& Kim, J. (2005). Effect Sizes for Research. A Broad Practical Approach. Mahwah: Lawrence Erlbaum

Jaramillo, J. (2012). Instrumento de medición de resiliencia adolescente. Revista de la facultad de ciencias médicas Universidad de Cuenca, $30(1), 10-15$

Rojas, V. (2015). Filosofía para niños. Práctica educativa y contexto social. Bogotá, Colombia: Editorial Magisterio.

Sánchez-Meca, J., Marin-Martinez, F., \& López-López, J. (2011) Metaanálisis e intervención Psicosocial basada en la evidencia. Revista Psychosocial Intervention. Colegio Oficial de Psicólogos de Madrid, 2O(1), 95-101.

Suárez, T., González, B., \& Lara, P. (2016). Aproximaciones y experiencias pedagógicas de filosofía e infancia en Colombia. Praxis \& Saber, 7(15), 225- 247. https://doi.org/10.19053/22160159.v7.n15.2016.5730

Unidad para la atención y reparación integral a las víctimas. (2019). Reporte número de personas por ciclo vital. Recuperado de https://www. unidadvictimas.gov.co/es/registro-unico-de-victimas-ruv/37394

Zabala, H. (2014). El desarrollo de las habilidades de pensamiento en el programa de filosofía para niños. Espiral, revista de docencia e investigación, 4(1), 59-70. https://doi.org/10.15332/erdi.v4i1.551 